\title{
Vaccines against bacterial zoonoses
}

Bacterial zoonoses such as anthrax, typhus and plague, have historically caused devastating human disease and have the potential to do so again should the appropriate environmental and social conditions arise. Others, including campylobacteriosis and salmonellosis are very common worldwide; some, such as brucellosis and leptospirosis, are of major economic significance because of their wide distribution and impact on animal production and public health. Emerging problems include Lyme disease associated with Borrelia burgdorferi and related species in Europe and North America, tick-borne relapsing fever associated with Bor. hermsi in the USA, ehrlichiosis and the expanding range of disease syndromes caused by Bartonella spp in immunocompetent and immunodeficient individuals.

Because of the need for contact with the animal host, its products or an arthropod vector, most human zoonoses are occupational, recreational or travelassociated. However, some are important for a more sinister reason: because of their high infectivity and ability to establish incapacitating or lethal infections from small inocula, some zoonotic agents are potentially useful as biological warfare agents. Indeed some, such as Bacillus anthracis, Burkholderia mallei, Francisella tularensis and Yersinia pestis, have been used for this purpose. Recognition of the inadequacies of currently available vaccines in the context of biological warfare has highlighted the need for new developments.

Vaccines are currently available for the prevention of anthrax, brucellosis, leptospirosis, plague, tularaemia, typhus and Q fever. Some of these have a very limited availability outside certain geographical areas and few are licensed in developed countries. The safety and efficacy of some of these products is also in question. Research on vaccines for zoonotic disease has been fairly limited, but the development of molecular cloning techniques has made possible the large-scale production of purified antigens and attenuated strains with defined genetic modifications.

Two types of anthrax vaccine for human use are available in the UK and USA. Both are based on partially purified protective antigen (PA) of the $B$. anthracis toxin complex adsorbed on to an aluminium adjuvant [1]. A live vaccine strain derived from the Sterne attenuated strain has been used in Russia and other countries of the former USSR [2]. The PA subunit vaccines are used to protect occupationally exposed groups and are evidently safe although transient adverse reactions seem frequent. Animal studies and epidemiological data provide circumstantial evidence of protection against respiratory and cutaneous anthrax in employees in high risk industries [3]. However, direct evidence of protective efficacy in man against aerosol challenge is lacking and experiments in animals suggest that protection against different $B$. anthracis strains may not be uniform [4]. The efficacy of this type of vaccine may be inherently less than that of live vaccines [2], but there would be considerable resistance to using the Russian vaccine in Western countries because of concerns over residual virulence and reactogenicity. Alternative options are limited. The key factors in the pathogenesis of anthrax are the production by the organism of the anti-phagocytic poly-D-glutamic acid capsule and the toxin complex. The latter comprises an oedema factor with adenyl cyclase activity [5], and a lethal factor that has metalloprotease activity and probably targets a macrophage subcellular protein [6]. Both toxins use PA for cell attachment and uptake, so that antibody to PA will neutralise both. Developments of highly purified PA for formulation with new adjuvants [7], or delivery of the antigen in vivo either in a 'designer' attenuated strain with defined genetic modifications or in a heterologous vector, have been effective. PA emulsified with monophosphoryl lipid A was more protective than PA with aluminium adjuvant [7] and cloning of the PA gene into an Aro-deletion mutant of Salmonella enterica serotype Typhimurium produced an expression system that stimulated immunity in mice to challenge by various routes, including respiratory aerosol exposure [8]. It is not yet known if these new vaccines will answer the need for a readily administered, low reactogenicity vaccine that will protect against all forms of anthrax.

Several vaccines protect against human brucellosis. The attenuated strains Brucella abortus $\mathrm{S} 19$ and $\mathrm{Br}$. melitensis $\operatorname{Rev} 1$ retain virulence for man and are unsuitable, but a variant of S19, Br. abortus S19-BA, has been used in the former USSR to protect occupationally exposed groups. Efficacy is limited and annual re-vaccination is needed. Adverse reactions are reported to be mild provided that the vaccine is administered by intradermal injection or skin scarification and not subcutaneously and that the recipients have not been sensitised. Another attenuated strain, $B r$. abortus $104 \mathrm{M}$, is administered in China for similar purposes by the skin scarification and aerosol routes. 
Neither of these vaccines would meet Western requirements for safety and efficacy.

A non-living vaccine with a phenol-insoluble peptidoglycan fraction [9] is claimed to stimulate a basic immunity to be reinforced by subclinical infection. Although of low reactogenicity, infections occurred in some recipients and its efficacy remains questionable. A protein-polysaccharide complex derived from smooth phase Brucella strains by mild acid hydrolysis is reported to be as protective as the $\mathrm{Br}$. abortus 19BA vaccine, but less reactogenic, at least in individuals not sensitised by previous infection [10].

Current strategies for the development of improved vaccines against human brucellosis centre around the development of attenuated strains with defined genetic modifications. The RB 51 strain, which was developed for the immunisation of cattle [11], is unlikely to be useful in man as it is no more protective than $\mathrm{S} 19$ and its human pathogenicity is uncertain. Equivalent $\mathrm{O}$ chain deficient mutants of $\mathrm{Br}$. melitensis or Br. suis may be worth examining but the most promising trend is the development of defined metabolic derivatives of virulent strains such as purE mutants [12, 13]. Because of the need to stimulate effective cell-mediated responses, subunit vaccines are less promising unless incorporating a delivery system or adjuvant capable of triggering Th1 responses. Fusion proteins incorporating the L7/L12 ribosomal proteins trigger antibody and cell-mediated responses and protection against challenge in mice [14]. They may have a future as components of human vaccines, possibly with detoxified lipopolysaccharide conjugates [15].

Current whole-cell plague vaccines stimulate immunity against bubonic plague, but are probably ineffective against the pneumonic disease [16, 17]. Subunit vaccines based on the fraction 1 and $\mathrm{V}$ antigens, either as purified proteins or fusion proteins, are effective against respiratory and parenteral challenge in animal models [18, 19]. Expression of these antigens in heterologous live vectors such as Aromutants of salmonella also stimulates good protection [20] although attenuation may not be complete [21]. These vaccines have yet to be evaluated in clinical trials.

Vaccines against Bor. burgdorferi, based on the outer surface protein Osp A [22], are in clinical trial in the USA. As most infections in North America are caused by Bor. burgdorferi sensu stricto antigenic variation is not a problem, but in Europe infections are also caused by Bor. afzeli, Bor. garini and unclassified species, in which the Osp A protein varies. Vaccines for Europe will have to be based on a cocktail of antigens; emphasis is being placed on the Osp B, Osp C, OspE and OspF proteins [23]. Such vaccines will probably include tick-borne fever antigens.
There seems much less interest in developing vaccines against Bor. hermsi and other relapsing fever spirochaetes even though these can cause lethal infections. This presumably reflects the perceived marketability of these vaccines, although relapsing fever vaccines would also be technically more difficult to develop because of antigenic variation in vivo.

A vaccine against tularaemia based on the LVS strain [24] is effective against the systemic as well as the localised oculo-glandular form of the disease, provided that the challenge is within the protection threshold. Immunity depends on a humoral response to the lipopolysaccharide and, possibly, a cell-mediated response to outer-membrane or other proteins [25]. Subunit vaccines may be feasible but the Fop A protein expressed in a salmonella vector was not protective [25].

A killed whole-cell vaccine based on phase 1 organisms is efficacious against occupational infection with $Q$ fever [26]. An acid extracted subunit vaccine has also been developed [27], and a live attenuated strain (M44), producing predominantly phase 2 organisms, has been used in the former USSR [28].

Vaccines prepared from killed yolk sac-grown organisms are available on a local basis for typhus and the spotted fevers, but little work is being done on other rickettsial infections. Some of these are clearly emerging as significant problems and more emphasis on vaccine development would seem justified. Orienta (Rickettsia) tsutsugamushi is still a problem in some areas, but the wide range of serotypes makes vaccine development difficult.

Vaccines against leptospirosis are widely used in dogs, cattle, pigs and sheep and appear to be protective. Equivalent vaccines for protection against human disease are available in only a few localities, although recreational exposure through water sports is an increasing problem. The immunity stimulated by killed whole-cell vaccines is serotype-specific and some, preparations may have to contain seven or more serotypes to cover locally prevalent strains [29]. The variety of potential serotypes to be covered complicates formulation, but new approaches, such as nucleic acid vaccines, may overcome this problem.

Considerable progress is being made in the development of vaccines against some zoonoses. However, there is less activity against diseases that are perceived solely as Third World or minority problems. Economic factors are likely to continue to regulate progress in this area.

M. J. CORBEL

Division of Bacteriology, National Institute for Biological Standards and Control, Potters Bar EN4 3QG. 


\section{References}

1. Hambleton P, Turnbull PCB. Anthrax vaccine development: a continuing story. In: Mizrahi A (ed) Bacterial vaccines. $A d v$ Biotechnol Processes 1990; 13: 105-122.

2. Shlyakov EN, Rubinstein E. Human live anthrax vaccine in the former USSR. Vaccine 1994: 12: 727-730.

3. Brachman PS, Gold H, Plotkin SA, Fekerty FR, Werrin M, Ingraham NR. Field evaluation of a human anthrax vaccine. Am J Public Health 1962; 52: 632-645.

4. Ivins BE, Fellows PF, Nelson GO. Efficacy of a standard human anthrax vaccine against Bacillus anthracis spore challenge in guinea-pigs. Vaccine 1994; 12: 872-874.

5. Leppla SH. Bacillus anthracis calmodulin-dependent adenylate cyclase: chemical and enzymatic properties and interactions with eukaryotic cells. In: Greengard P (ed) Advances in cyclic nucleotide and protein phosphorylation research. Orlando, Academic Press. 1984: 103-116.

6. Klimpel KR, Arora N, Leppla SH. Anthrax toxin lethal factor contains a zinc metalloprotease consensus sequence which is required for lethal toxin activity. Mol Microbiol 1994; 13: 1093-1100.

7. Ivins B, Fellows P, Pitt L et al. Experimental anthrax vaccines: efficacy of adjuvants combined with protective antigen against an aerosol Bacillus anthracis spore challenge in guinea pigs. Vaccine 1995; 13: 1779-1794

8. Coulson NM, Fulop M, Titball RW. Bacillus anthracis protective antigen expressed in Salmonella typhimurium SL 3261 , affords protection against spore challenge. Vaccine 1994; 12: 1395-1401.

9. Lopez Merino A, Asselineau J, Serre A, Roux J, Bascoul S, Lacave C. Immunization by an insoluble fraction extracted from Brucella melitensis: immunological and chemical characterization of the active substances. Infect Immun 1976; 13: $311-321$.

10. Dranovskaya EA. New approaches to brucellosis vaccination of people with high risk of infection. In: Tumbay E, Hilmi S, Ang $\mathrm{O}$ (eds) Brucella and brucellosis in man and animals. Turkish Microbiology Society 1991; 16: 87-100. Ege University Press, Izmir.

11. Schurig GG, Roop RM, Bagchi T, Boyle S, Buhrman D, Sriranganathan N. Biological properties of RB51. A stable strain of Brucella abortus. Vet Microbiol 1991; 28: 171-188.

12. Drazek ES, Young HH, Crawford RM, Hadfield TL, Hoover $\mathrm{DL}$, Warren RL. Deletion of pur $E$ attenuates Brucella melitensis $16 \mathrm{M}$ for growth in human monocyte-derived macrophages. Infect Immun 1995; 63: 3277-3301.

13. Cheville NF, Olsen SC, Jensen $\mathrm{AE}$ et al. Bacterial persistence and immunity in goats vaccinated with a pur $E$ deletion mutant or the parental $16 \mathrm{M}$ strain of Brucella melitensis. Infect Immun 1996; 64: 2431-2439.

14. Oliveira SC, Splitter GA. Immunization of mice with recom- binant $\mathrm{L} 7 / \mathrm{L} 12$ ribosomal protein confers protection against Brucella abortus infection. Vaccine 1996; 14: 959-962.

15. Jacques I, Olivier-Bernardin V, Dubray G. Induction of antibody and protective responses in mice by Brucella $\mathrm{O}$ polysaccharide-BSA conjugate. Vaccine 1991; 9: 896-900.

16. Russel P, Eley SM, Hibbs SE, Manchee RJ, Stagg AJ, Titball RW. A comparison of Plague Vaccine, USP and EV76 vaccine induced protection against Yersinia pestis in a murine model. Vaccine 1995; 13: 1551-1556.

17. Meyer KF. Effectiveness of live or killed plague vaccines in man. Bull World Health Organ 1970; 42: 653-666.

18. Andrews GP, Heath DG, Anderson GW, Welkos SL, Friedlander AM. Fraction-1 capsular antigen (F1) purification from Yersinia pestis $\mathrm{CO} 92$ and from an Escherichia coli recombinant strain and efficacy against lethal plague challenge. Infect Immun 1996; 64: 2180-2187.

19. Leary SEC, Williamson ED, Griffin KF, Russell P, Eley SM, Titball RW. Active immunization with recombinant $\mathrm{V}$ antigen for Yersinia pestis protects mice against plague. Infect Immun 1995; 63: 2854-2858.

20. Oyston PCF, Williamson ED, Leary SEC, Eley SM, Griffin KF, Titball RW. Immunization with live recombinant Salmonella typhimurium aroA producing $\mathrm{F} 1$ antigen protects against plague. Infect Immun 1995; 63: 563-568.

21. Oyston PCF, Russell P, Williamson ED, Titball RW. An aro $A$ mutant of Yersinia pestis is attenuated in guinea-pigs, but virulent in mice. Microbiology 1996; 142: 1847-1853.

22. Keller D, Koster FT, Marks DH, Hosbach P, Erdile LF, Mays JP. Safety and immunogenicity of a recombinant outer surface protein A Lyme vaccine. JAMA 1994; 271: 1764-1768.

23. Eldelman R. The Sixth International Conference on Lyme borreliosis: progress on the development of Lyme disease vaccines, Vaccine 1995; 13: 133-135.

24. Hornick RB, Eigelsbach HT. Aerogenic immunization of man with live tularaemia vaccine. Bact Rev 1966; 30: 532-538.

25. Fulop M, Manchee R, Titball R. Role of lipopolysaccharide and a major outer membrane protein from Francisella tularensis in the induction of immunity against tularaemia. Vaccine 1995; 13: 1220-1225.

26. Ackland JR, Worswick DA, Marmion BP. Vaccine prophylaxis of $\mathrm{Q}$ fever. A follow-up study of the efficacy of Q-Vac (CSL) 1985-1990. Med J Aust 1994; 160: 704-708.

27. Kaźar J, Brezhina R, Palanová A, Tvrdá B, Schramek Ś Immunogenicity and reactogenicity of a $Q$ fever chemovaccine in persons professionally exposed to $Q$ fever in Czechoslovakia. Bull World Health Organ 1982; 60: 389-394.

28. Genig VA. Experience on mass immunization of human beings with the M-44 live vaccine against Q-fever. Report 2. Skin and oral routes of immunization. Vopr Virusol 1965; 6: 703-707.

29. Chen TZ. Development and present status of leptospiral vaccine and technology of production of the vaccine in China. Ann Immunol Hung 1986; 26: 125-151. 